Paolo Ciatti

\title{
A Clifford algebra approach to simple Lie algebras of real rank two, II: The $G_{2}$ case
}

Received: November 11, 1999

Published online: March 19, 2002 - (c) Springer-Verlag 2002

\section{Introduction}

In a couple of papers, Cowling, Dooley, Korányi, and Ricci [3,4] proposed an approach to real rank one simple Lie algebras which is based on the observation, due to Korányi [7], that the nilpotent subalgebras obtained in the Iwasawa decomposition of these simple Lie algebras are generalized Heisenberg algebra (see [6]). This simply means that the orthogonal complement of the centre of an Iwasawa algebra is a module of the Clifford algebra built on the centre. Recently the author [2] succeded in extending this approach to simple Lie algebras with root system $A_{2}$. Here, we study with the same technique the exceptional simple Lie algebras with root system $G_{2}$, showing that the same Clifford algebra approach works also in this exceptional context despite the complications due to the richer structure of these algebras.

Here is the outline of the paper. In Sect. 2 we briefly recall the structure of the root sistem $G_{2}$.

In Sect. 3 we study the subalgebras $\mathfrak{n}$ and $\mathfrak{m}$ obtained by performing the Iwasawa decomposition $\mathfrak{k} \oplus \mathfrak{a} \oplus \mathfrak{n}$ of the Lie algebra. In particular, in Subsect. 3.1 we decompose $\mathfrak{n}$ into the semidirect product of a generalized Heisenberg algebra $\mathfrak{n}_{0}$ and the root space corresponding to the shortest of the simple roots, say $\alpha$. In Subsect. 3.2 we study the action of the rank one subalgebra $\mathfrak{g}_{(\alpha)}$ generated by the root spaces $\mathfrak{g}_{\alpha}$ and $\mathfrak{g}_{-\alpha}$ on $\mathfrak{n}_{0}$. Finally in Subsect. 3.3 we examine the structure of $\mathfrak{m}$, the centralizer of $\mathfrak{a}$ in $\mathfrak{k}$, and its action on $\mathfrak{n}$. We use this analysis to show that all root spaces must have the same multiplicities and that these may only be 1 , or 2.

In Sect. 4 we construct a skew-symmetric algebra $\mathfrak{g}$ of dimension 14 . We start in Subsect. 4.1 considering two generalized Heisenberg algebras which correspond to $\mathfrak{n}_{0}$ and $\theta \mathfrak{n}_{0}$. Then in Subsect. 4.2 we build the algebra of deriva-

P. Ciatti: Dipartimento di Metodi matematici, Universitá di Padova, Via Belzoni, 7, 35131, Padova, Italy 
tions of $\mathfrak{n}_{0}$ corresponding to $\mathfrak{g}_{(\alpha)}$. Eventually in Subsect. 4.3 we obtain the full algebra $\mathfrak{g}$.

In Sect. 6 we show that the bracket of $\mathfrak{g}$ satisfies the Jacobi identity, proving that $\mathfrak{g}$ is a Lie algebra.

Finally, in Sect. 7 we prove that any simple Lie algebra with root system $G_{2}$ and dimension 28 is the complexification of $\mathfrak{g}$.

\section{The root system $G_{2}$}

Denote by $\theta$ the Cartan involution and by $B: \mathfrak{g} \times \mathfrak{g} \rightarrow \mathbb{R}$ the Killing form of $\mathfrak{g}$, and set

$$
\langle X, Y\rangle=-c B(X, \theta Y), \quad \forall X, Y \in \mathfrak{g}
$$

where $c$ is a positive constant to be fixed later. It is clear that $\langle\cdot, \cdot\rangle$ is an inner product. Note the identity

$$
\langle[X, Y], Z\rangle=\langle X,[\theta Y, Z]\rangle,
$$

which is equivalent to the invariance of the Killing form.

Denote by $\Delta$ the set of restricted roots, that is $G_{2}$, by $\Delta_{+}$the set of positive roots, and by $\alpha$ and $\beta$ the simple roots. Then

$$
\begin{gathered}
\Delta_{+}=\{\alpha, \beta, \alpha+\beta, 2 \alpha+\beta, 3 \alpha+\beta, 3 \alpha+2 \beta\}, \\
(\alpha \mid \beta)=-\frac{1}{2}(\beta \mid \beta),
\end{gathered}
$$

and the highest root $[5, \mathrm{p} .475]$ is

$$
\omega=3 \alpha+2 \beta .
$$

We choose $c$ in $\langle\cdot, \cdot\rangle$ so that

$$
(\beta \mid \beta)=(3 \alpha+\beta \mid 3 \alpha+\beta)=(\omega \mid \omega)=2,
$$

it follows that

$$
(\alpha \mid \beta)=-1, \quad(\alpha \mid \alpha)=\frac{2}{3}
$$

and

$$
(\omega \mid \beta)=(\omega \mid \alpha+\beta)=(\omega \mid 2 \alpha+\beta)=(\omega \mid 3 \alpha+\beta)=1 .
$$




\section{The structure of the algebra}

\subsection{The Clifford structure}

Proposition 3.1.1. Let

$$
\mathfrak{v}=\mathfrak{g}_{\beta} \oplus \mathfrak{g}_{\alpha+\beta} \oplus \mathfrak{g}_{\alpha+2 \beta} \oplus \mathfrak{g}_{\alpha+3 \beta} .
$$

For $Z \in \mathfrak{g}_{\omega}$ define the linear operator $J_{Z}: \mathfrak{v} \rightarrow \mathfrak{v}$ by

$$
J_{Z} X=[Z, \theta X],
$$

with $X \in \mathfrak{v}$. Then for all $X, Y \in \mathfrak{v}$

$$
\left[X, J_{Z} X\right]=\|X\|^{2} Z,
$$

and

$$
J_{Z}^{2} X=-\|Z\|^{2} X
$$

that is, $\mathfrak{v}$ is a Clifford module of the Clifford algebra $\mathcal{C}\left(d_{\omega}\right)$, where $d_{\omega}$ denote the dimension of $\mathfrak{g}_{\omega}$.

Proof. The statement follows from the Jacobi identity and (2.1.2), using

$$
[\theta X, X]=\|X\|^{2} H_{\gamma},
$$

which holds for $X \in \mathfrak{g}_{\gamma}$ [5, p. 94].

Remark. Since $J_{Z}$ is skew-symmetric, $n_{0} \equiv \mathfrak{v} \oplus \mathfrak{g}_{\omega}$ endowed with $\langle\cdot, \cdot\rangle$ is by (3.1.2) a generalized Heisenberg algebra with centre $\mathfrak{g}_{\omega}$ ([6] and [7]).

Corollary 3.1.2. (1) $d_{\omega} \leq d_{\alpha+\beta}=d_{2 \alpha+\beta}=d_{\alpha}$;

(2) $d_{\omega}=d_{\beta}=d_{3 \alpha+\beta} \in\{1,2,4,8\}$.

Proof. Since by (3.1.4) the restrictions of $J_{Z}$ to $\mathfrak{g}_{\beta}$ and $\mathfrak{g}_{\alpha+\beta}$ are, for $Z \neq 0$, invertible maps onto $\mathfrak{g}_{3 \alpha+\beta}$ and $\mathfrak{g}_{2 \alpha+\beta}$, respectively, $d_{\beta}=d_{3 \alpha+\beta}$ and $d_{\alpha+\beta}=d_{2 \alpha+\beta}$.

By (3.1.1) $d_{\beta} \geq d_{\omega}$ and $d_{\alpha+\beta} \geq d_{\omega}$. Let $X \in \mathfrak{g}_{\beta}$, take a unit $Y \in \mathfrak{g}_{3 \alpha+\beta}$, then $[X, Y] \in \mathfrak{g}_{\omega}$. By Jacobi, since $\omega-3 \alpha-\beta \notin \Delta$, we find

$$
[\theta Y,[Y, X]]=-X
$$

Hence, $d_{\omega} \geq d_{\beta}$, from which it follows $d_{\omega}=d_{\beta}$. Similarly taking a unit $Y \in \mathfrak{g}_{\beta}$ we prove that $d_{\alpha}=d_{\alpha+\beta}$, providing (1).

(2) From the proof of Proposition 3.1.1 we see that $\mathfrak{g}_{\beta} \oplus \mathfrak{g}_{3 \alpha+\beta}$ is a module of $\mathcal{C}\left(d_{\omega}\right)$, which by (1) has dimension $2 d_{\omega}$. By the classification of Clifford algebras the algebra $\mathcal{C}\left(d_{\omega}\right)$ has a module of dimension $2 d_{\omega}$ only when $d_{\omega} \in\{1,2,4,8\}$, and this provides the statement. 


\subsection{The action of $\mathfrak{g}_{(\alpha)}$}

Lemma 3.2.1. Let $S \in \mathfrak{g}_{\alpha}$ and $X \in \mathfrak{g}_{\beta}$, then

$$
\begin{gathered}
{[\theta S,[S, X]]=-\|S\|^{2} X,} \\
{[\theta S,[S,[S, X]]]=-\frac{4}{3}\|S\|^{2}[S, X],}
\end{gathered}
$$

and

$$
[\theta S,[S,[S,[S, X]]]]=-\|S\|^{2}[S,[S, X]] .
$$

In particular, if both $S$ and $X$ are non-zero, then

$$
[S, X] \neq 0, \quad[S,[S, X]] \neq 0,[S,[S,[S, X]]] \neq 0 .
$$

Proof. Since $\beta-\alpha \notin \Delta$, the Jacobi identity implies

$$
[\theta S,[S, X]]=[X,[S, \theta S]]=\beta([\theta S, S]) X=(\beta \mid \alpha)\|S\|^{2} X=-\|S\|^{2} X \neq 0 .
$$

Using (3.2.1) and the Jacobi identity we get

$$
\begin{aligned}
{[\theta S,[S,[S, X]]] } & =[S,[\theta S,[S, X]]]+[[S, X],[S, \theta S]] \\
& =-\|S\|^{2}[S, X]+(\alpha+\beta)([\theta S, S])[S, X] \\
& =-\frac{4}{3}\|S\|^{2}[S, X] \neq 0 .
\end{aligned}
$$

Similarly, from (3.2.2) we find

$$
\begin{aligned}
{[\theta S,[S,[S,[S, X]]]] } & =[S,[\theta S,[S,[S, X]]]]+[[S,[S, X]],[S, \theta S]] \\
& =-\frac{4}{3}\|S\|^{2}[S,[S, X]]+(2 \alpha+\beta)([\theta S, S])[S,[S, X]] \\
& =-\|S\|^{2}[S,[S, X]] \neq 0 .
\end{aligned}
$$

Proposition 3.2.2. $d_{\omega} \in\{1,2\}$.

Proof. Assume $d_{\omega}=d \in\{4,8\}$, and let $\left\{Z_{1}, Z_{2}, \ldots, Z_{d_{\omega}}\right\}$ be an orthonormal basis of $\mathfrak{g}_{\omega}$. Set

$$
\epsilon=J_{1} J_{2} \ldots J_{d},
$$

then $\epsilon$ is symmetric and $\epsilon^{2}=1$. Therefore, $\epsilon$ has eigenvalues \pm 1 . Let $V \in \mathfrak{v} \backslash\{0\}$ and $\epsilon V=V, V$ has a non-zero component in one of the root spaces in $\mathfrak{v}$, say $X \in \mathfrak{g}_{\gamma}$. It is clear that $\left\{X, J_{1} J_{2} X, \ldots, J_{1} J_{d} X\right\}$ and $\left\{J_{1} X, J_{2} X, \ldots, J_{d} X\right\}$ span $\mathfrak{g}_{\gamma}$ and $\mathfrak{g}_{\omega-\gamma}$. Since $\epsilon J_{k}=-J_{k} \epsilon$, the restriction of $\epsilon$ to $\mathfrak{g}_{\gamma}$ is the identity and the restriction to $\mathfrak{g}_{\omega-\gamma}$ is minus the identity. Hence, the component of $V$ in $\mathfrak{g}_{\omega-\gamma}$ is 
zero. It is not restrictive to assume that $\epsilon$ is the identity on $\mathfrak{g}_{\alpha+\beta}$ and minus the identity on $\mathfrak{g}_{2 \alpha+\beta}$.

Take a unit vector $S \in \mathfrak{g}_{\alpha}$. Since ad $S$ commutes on $\mathfrak{g}_{\alpha+\beta} \oplus \mathfrak{g}_{2 \alpha+\beta}$ with $J_{i} J_{k}$, it also commutes with $\epsilon$. Hence, for all $Y \in \mathfrak{g}_{\alpha+\beta}$,

$$
\epsilon[S, Y]=[S, \epsilon Y]=[S, Y] .
$$

This provides a contradiction, since $[S, Y] \neq 0$ if $Y=\left[S, Y^{\prime}\right]$ with $Y^{\prime} \in \mathfrak{g}_{\beta} \backslash\{0\}$ by (3.2.2).

\subsection{The structure of $\mathfrak{m}$}

In Subsect. 3.2 we established $d_{\omega} \in\{1,2\}$. Now exploiting the structure of $\mathfrak{m}$, the centralizer of $\mathfrak{a}$ in $\mathfrak{k}$, we shall determine the allowed multiplicities of the roots. Let $\gamma \in \Delta$, we denote by $\mathfrak{g}_{(\gamma)}$ the rank one subalgebra generated by $\mathfrak{g}_{\gamma}$ and $\mathfrak{g}_{-\gamma}$ and set

$$
\mathfrak{m}_{\gamma}=\mathfrak{m} \cap \mathfrak{g}_{\gamma} .
$$

We collect in the following lemma some results more or less known, whose proof can, however, be found in [2].

Lemma 3.3.0. Suppose $\gamma, \delta \in \Delta$ and $2 \gamma \notin \Delta$, and fix an orthonormal basis of $\mathfrak{g}_{\gamma}$ $\left\{X_{1}, \ldots, X_{d_{\gamma}}\right\}$.

(1)

$$
\mathfrak{m}_{\gamma}=\operatorname{span}\left\{\left[X_{i}, \theta X_{j}\right]: 1 \leq i<j \leq d_{\gamma}\right\}
$$

(2)

$$
\left[\left[X_{i}, \theta X_{j}\right], X_{i}\right]=(\gamma \mid \gamma) X_{j} \quad \text { if } i \neq j ;
$$

(3)

$$
\left[\left[X_{i}, \theta X_{j}\right], X_{k}\right]=0 \quad \text { if } i \neq j, i \neq k, \text { and } j \neq k
$$

(4) $\mathfrak{m}_{\gamma} \simeq \mathfrak{s o}\left(d_{\gamma}\right)$;

(5) $\mathfrak{m}_{\gamma} \cap \mathfrak{m}_{\delta}$ is an ideal in $\mathfrak{m}_{\gamma}$ and $\mathfrak{m}_{\delta}$;

(6) if the action of $\mathfrak{m}_{\delta}$ on $\mathfrak{g}_{\gamma}$ is trivial, then $\mathfrak{m}_{\gamma} \cap \mathfrak{m}_{\delta}=\{0\}$. When $d_{\gamma} \geq 3$, if $\mathfrak{m}_{\gamma} \cap \mathfrak{m}_{\delta}=\{0\}$, the action of $\mathfrak{m}_{\delta}$ on $\mathfrak{g}_{\gamma}$ is trivial.

Proof. (1) follows from Proposition 2.3 of [2]. (2) is Formula (8) of [2]. (3) is Formula (9) of [2]. (4) is Corollary 2.5 of [2]. (5) is obvious. (6) is Proposition 2.8 of [2].

Proposition 3.3.1. Suppose $d_{\omega}=2$.

(1) Let $\gamma \in\{\beta, \alpha+\beta, 2 \alpha+\beta, 3 \alpha+\beta\}$, then $\mathfrak{m}_{\omega} \cap \mathfrak{m}_{\gamma}=\{0\}$;

(2) $d_{\gamma}=2$ for all $\gamma \in \Delta$;

(3) $\mathfrak{m}=\mathfrak{m}_{\omega} \oplus \mathfrak{m}_{\alpha} \simeq \mathfrak{s o}(2) \oplus \mathfrak{s o}(2)$. 
Proof. Since $d_{\omega} \geq 2, \mathfrak{m}_{\omega}$ is non-trivial. By Corollary 3.1.2 $d_{\gamma} \geq 2$.

(1) Suppose by contradiction that $\mathfrak{m}_{\omega} \cap \mathfrak{m}_{\gamma} \neq\{0\}$. Being $\operatorname{dim} \mathfrak{m}_{\omega}=1$, it follows by Lemma 3.3.0 (5) that $\mathfrak{m}_{\omega}$ is a one-dimensional ideal in $\mathfrak{m}_{\gamma}$. Since, by Lemma 3.3.0 (4), $\mathfrak{m}_{\gamma}$ is isomorphic to $\mathfrak{s o}\left(d_{\gamma}\right)$, which has a one-dimensional ideal only for $d_{\gamma}=2$, we obtain $\mathfrak{m}_{\gamma}=\mathfrak{m}_{\omega}$. Therefore, if $\left\{Z_{1}, Z_{2}\right\}$ is an orthonormal basis of $\mathfrak{g}_{\omega}$ and $X \in \mathfrak{g}_{\gamma}$ a unit vector,

$$
\left[Z_{1}, \theta Z_{2}\right]=c\left[X, \theta J_{1} J_{2} X\right], \quad \text { with } c \in \mathbb{R} .
$$

Applying both sides of (3.3.1) to $Z_{1}$, by Jacobi we get, using Lemma 3.3.0 (2), (3.3.1), and (3.1.2),

$$
2 Z_{2}=c\left[\left[X, \theta J_{1} J_{2} X\right], Z_{1}\right]=c\left[\left[Z_{1}, \theta J_{1} J_{2} X\right], X\right]=c\left[J_{1}^{2} J_{2} X, X\right]=c Z_{2},
$$

whence $c=2$. On the other hand, (3.3.1) applied to $X$ gives, by Lemma 3.3.0 (2),

$$
J_{1} J_{2} X=\left[\left[\theta Z_{1}, Z_{2}\right], X\right]=2\left[\left[\theta X, J_{1} J_{2} X\right], X\right]=2 \gamma([\theta X, X]) J_{1} J_{2} X,
$$

which implies $(\gamma \mid \gamma)=1 / 2$, yielding a contradiction.

(2) By Corollary 3.1.2 (2) we consider $\gamma \in\{\alpha+\beta, 2 \alpha+\beta\}$. Since $\mathfrak{m}_{\omega} \cap \mathfrak{m}_{\gamma}=\{0\}$, if it were $d_{\gamma} \geq 3$ the action of $\mathfrak{m}_{\omega}$ on $\mathfrak{g}_{\gamma}$ would be trivial by Lemma 3.3.0 (6), providing a contradiction being $\left(\operatorname{ad}\left[Z_{1}, \theta Z_{2}\right]\right) X=J_{1} J_{2} X$ for all pairs of orthogonal vectors $Z_{1}$ and $Z_{2}$ in $\mathfrak{g}_{\omega}$ and all $X \in \mathfrak{g}_{\gamma}$. Hence, $d_{\gamma}=2$.

Lemma 3.3.2. If $d_{\omega}=1$, then $d_{\gamma}=1$ for all $\gamma \in \Delta$.

Proof. By Corollary 3.1.2 (2) $d_{\beta}=d_{3 \alpha+\beta}=1$. We prove that $d \equiv d_{\alpha+\beta}=d_{\alpha}=$ $d_{2 \alpha+\beta}=1$. Fix an arthonormal basis $\left\{X_{1}, \ldots, X_{d}\right\}$ of $\mathfrak{g}_{\alpha+\beta}$ and a unit $Z \in \mathfrak{g}_{\omega}$. Then set $Y_{i}=J_{Z} X_{i}$, it follows that $\left\{Y_{1}, \ldots, Y_{d}\right\}$ is an orthonormal basis of $\mathfrak{g}_{2 \alpha+\beta}$,

$$
\left[X_{i}, Y_{k}\right]=\delta_{i k} Z \text { and }\left[X_{i}, \theta X_{k}\right]=\left[Y_{i}, \theta Y_{k}\right],
$$

where $\delta_{i k}$ is the Kroeneker delta, the first relation is a consequence of (3.1.3), and the second is obtained using the Jacobi identity.

We prove the proposition deducing from $d_{\alpha} \geq 2$ that for $i \neq j$

$$
\left[X_{i}, \theta Y_{i}\right], \quad\left[X_{j}, \theta Y_{j}\right], \text { and }\left[X_{i}, \theta Y_{j}\right]
$$

are linear independent vectors in $\mathfrak{g}_{\alpha}$, contradicting Corollary 3.1.2 (1). The Jacobi identity and (2.1.0) provide

$$
\begin{aligned}
\left\langle\left[X_{j}, \theta Y_{i}\right],\left[X_{l}, \theta Y_{k}\right]\right\rangle & =\left\langle X_{j},\left[Y_{i},\left[X_{l}, \theta Y_{k}\right]\right]\right\rangle \\
& =\left\langle X_{j},\left[X_{l},\left[Y_{i}, \theta Y_{k}\right]\right]\right\rangle+\left\langle X_{j},\left[\theta Y_{k},\left[X_{l}, Y_{i}\right]\right]\right\rangle .
\end{aligned}
$$

This relation for $i=j=k=l$ yields

$$
\left\|\left[X_{i}, \theta Y_{i}\right]\right\|^{2}=\frac{1}{3}\left\|X_{i}\right\|^{2}+\left\langle X_{i},\left[\theta Y_{i}, Z\right]\right\rangle=\frac{4}{3},
$$

and also, using the first relation in (3.3.2),

$$
\left\|\left[X_{i}, \theta Y_{j}\right]\right\|^{2}=-(\alpha+\beta)\left(\left[Y_{j}, \theta Y_{j}\right]\right)\left\|X_{i}\right\|^{2}=\frac{1}{3} .
$$


Moreover, from the relations (3.3.2) and Lemma 3.3.0 (2) we get for $i \neq j$

$$
\left\langle\left[X_{i}, \theta Y_{i}\right],\left[X_{j}, \theta Y_{j}\right]\right\rangle=\left\langle X_{i},\left[X_{j},\left[Y_{i}, \theta Y_{j}\right]\right]\right\rangle+\left\langle X_{i},\left[\theta Y_{j},\left[X_{j}, Y_{i}\right]\right]\right\rangle=\frac{1}{3},
$$

which together with (3.3.3) implies, by the converse of the Schwarz inequality, that $\left[X_{i}, \theta Y_{i}\right]$ and $\left[X_{j}, \theta Y_{j}\right]$ are linearly independent. Finally, by (3.3.2) we find for $i \neq l \neq k \neq i$

$$
\begin{aligned}
\left\langle\left[X_{i}, \theta Y_{i}\right],\left[X_{l}, \theta Y_{k}\right]\right\rangle & =\left\langle X_{i},\left[X_{l},\left[Y_{i}, \theta Y_{k}\right]\right]\right\rangle+\left\langle X_{i},\left[\theta Y_{k},\left[X_{l}, Y_{i}\right]\right]\right\rangle \\
& =\left\langle X_{i},\left[X_{l},\left[X_{i}, \theta X_{k}\right]\right]\right\rangle=0 .
\end{aligned}
$$

This completes the proof.

Summarizing the above results we obtain:

Proposition 3.3.3. (1) There are two possible cases: all the roots have multiplicity 1 , and all the roots have multiplicity 2 .

(2) If $d_{\omega}=1$, then $\mathfrak{m}=\{0\}$. If $d_{\omega}=2$, then $\mathfrak{m}=\mathfrak{m}_{\omega} \oplus \mathfrak{m}_{\beta} \backsim \mathfrak{s o}(2) \oplus \mathfrak{s o}(2)$.

Lemma 3.3.4. Let $Z \in \mathfrak{g}_{\omega}, X \in \mathfrak{g}_{\beta}$, and $Y \in \mathfrak{g}_{\alpha+\beta}$ be unit vectors, then

$$
\left\|\left[\theta Y, J_{Z} Y\right]\right\|^{2}=\frac{4}{3}\|[\theta X, Y]\|^{2}=1 .
$$

Proof. The first relation is Formula (3.3.3) in the proof of Lemma 3.3.2, for the second we find,

$$
\begin{aligned}
\|[\theta X, Y]\|^{2} & =\langle[[\theta X, Y], X], Y\rangle \\
& =(\alpha+\beta)([\theta X, X])\|Y, Y\|^{2}=(\alpha+\beta \mid \beta)=1 .
\end{aligned}
$$

We denote by $M_{\gamma}$ the element of $\mathfrak{m}_{\gamma}$ normalized by the condition

$$
\left\|M_{\gamma}\right\|=(\gamma \mid \gamma)
$$

Lemma 3.3.5. Suppose $d_{\omega}=2$. Let $\gamma$ and $\gamma^{\prime}$ be orthogonal roots. For $\delta \in \Delta, M_{\delta}$ is given by

$$
M_{\delta}=\frac{(\delta \mid \gamma)}{(\gamma \mid \gamma)} M_{\gamma}+\frac{\left(\delta \mid \gamma^{\prime}\right)}{\left(\gamma^{\prime} \mid \gamma^{\prime}\right)} M_{\gamma^{\prime}}
$$

If $X \in \mathfrak{g}_{\gamma}$, then

$$
\left[M_{\delta}, X\right]=\frac{(\delta \mid \gamma)}{(\gamma \mid \gamma)}\left[M_{\gamma}, X\right] .
$$


Proof. The statement makes sense since for all $\gamma \in \Delta$ there are two roots orthogonal to $\gamma$. Moreover, $\gamma+\gamma^{\prime} \notin \Delta$. There are two orthogonal vectors $X_{1}$ and $X_{2}$ in $\mathfrak{g}_{\gamma}$ such that $M_{\gamma}=\left[X_{1}, \theta X_{2}\right]$. Using the Jacobi identity and (2.1.0) we find

$$
\left\langle M_{\gamma}, M_{\gamma^{\prime}}\right\rangle=\left\langle\left[X_{1}, \theta X_{2}\right], M_{\gamma^{\prime}}\right\rangle=\left\langle X_{1},\left[X_{2}, M_{\gamma^{\prime}}\right]\right\rangle=0 .
$$

since, being $\gamma+\gamma^{\prime} \notin \Delta,\left[X_{2}, M_{\gamma^{\prime}}\right]=0$. Therefore, by Lemma 3.3.0 (1) we obtain

$$
M_{\delta}=a M_{\gamma}+b M_{\gamma^{\prime}}, \quad \text { with } a, b \in \mathbb{R} .
$$

Now, (3.3.5) follows from

$$
\left\|M_{\delta}\right\|^{2}=(\delta \mid \delta)=\frac{(\delta \mid \gamma)^{2}}{(\gamma \mid \gamma)}+\frac{\left(\delta \mid \gamma^{\prime}\right)^{2}}{\left(\gamma^{\prime} \mid \gamma^{\prime}\right)},
$$

(3.3.4), and (3.3.7). Since $\left[M_{\gamma^{\prime}}, X\right]=0,(3.3 .6)$ is now an immediate consequence of (3.3.5).

Lemma 3.3.6. Let $Z \in \mathfrak{g}_{\omega}$ be a unit vector, and let $X \in \mathfrak{g}_{\beta}$ and $Y \in \mathfrak{g}_{\alpha+\beta}$,

$$
[\theta X, Y]=\left[J_{Z} X, \theta J_{Z} Y\right] .
$$

When $d=2$, if $Z_{1}, Z_{2} \in \mathfrak{g}_{\omega}$ is an orthonormal basis of $\mathfrak{g}_{\omega}$, then

$$
\left[J_{1} J_{2} X, \theta Y\right]=\left[\theta J_{1} J_{2} Y, X\right] .
$$

Proof. For (3.3.8), we have

$$
\left[J_{Z} X, \theta J_{Z} Y\right]=\left[J_{Z} X,[\theta Z, Y]\right]=\left[Y,\left[\theta Z, J_{Z} X\right]\right]=[\theta X, Y] .
$$

For (3.3.9), since $\omega \pm \alpha \notin \Delta$,

$$
0=\left[\left[Z_{1}, \theta Z_{2}\right],[X, \theta Y]\right]=\left[J_{1} J_{2} X, \theta Y\right]+\left[X, \theta J_{1} J_{2} Y\right] .
$$

\section{The construction of $\mathfrak{g}$}

\subsection{Construction of $\mathfrak{n}_{0+}$ and $\mathfrak{n}_{0-}$}

Let $\mathfrak{z}=\mathbb{R}$ with $\left\langle Z, Z^{\prime}\right\rangle_{\mathfrak{z}}=Z Z^{\prime}$. Take two irreducible modules $\left(\mathfrak{v}_{1}, J^{(1)}\right)$ and $\left(\mathfrak{v}_{2}, J^{(2)}\right)$ of $\mathcal{C}(1) \simeq \mathbb{C}$, where $\mathfrak{v}_{1} \simeq \mathfrak{v}_{2} \simeq \mathbb{R}^{2}$, and $J^{(i)}: \mathcal{C}(1) \rightarrow$ End $\left(\mathfrak{v}_{i}\right)$ for $i=1,2$. Endow $\mathfrak{v}_{1}$ and $\mathfrak{v}_{2}$ with the euclidean inner porduct of $\mathbb{R}^{2}\langle\cdot, \cdot\rangle_{i}, i=1,2$. The operators $J_{a}^{(1)}$ and $J_{a}^{(2)}$ are skew-symmetric with respect to these inner products for all $a \in \mathcal{C}(1)$. Define

$$
\mathfrak{n}_{0}=\mathfrak{v}_{1} \oplus \mathfrak{v}_{2} \oplus \mathfrak{z}\langle\cdot, \cdot\rangle=\langle\cdot, \cdot\rangle_{1} \oplus\langle\cdot, \cdot\rangle_{2} \oplus\langle\cdot, \cdot\rangle_{\mathfrak{z}},
$$

and equip $\left(\mathfrak{n}_{0},\langle\cdot, \cdot\rangle\right)$ with the corresponding structure of generalized Heisenberg algebra. Notice that this is just the Heisenberg algebra $\mathbb{H}_{2}$. Fix a unit vector $Z_{0} \in \mathfrak{z}$ 
and set $J=J_{Z_{0}}$. Fix moreover a unit vector $X_{0} \in \mathfrak{v}_{1}$ and a unit vector $Y_{0} \in \mathfrak{v}_{2}$, obtaining

$$
\mathfrak{v}_{1}=\operatorname{span}\left\{X_{0}, J X_{0}\right\} \mathfrak{v}_{2}=\operatorname{span}\left\{Y_{0}, J Y_{0}\right\}
$$

Put

$$
\mathfrak{v}_{11}=\mathbb{R} X_{0}, \quad \mathfrak{v}_{12}=\mathbb{R} J X_{0}, \quad \mathfrak{v}_{21}=\mathbb{R} Y_{0}, \quad \mathfrak{v}_{22}=\mathbb{R} J Y_{0}
$$

To complete the picture one needs another copy of $\mathfrak{n}_{0}$. Therefore, consider the cartesian product $\mathfrak{n}_{0} \times\{+,-\}$ and set

$$
\mathfrak{n}_{0+}=\mathfrak{n}_{0} \times\{+\}, \quad \mathfrak{n}_{0-}=\mathfrak{n}_{0} \times\{-\}, \mathfrak{n}_{0} \times\{+,-\}=\mathfrak{n}_{0+} \oplus \mathfrak{n}_{0-} .
$$

Correspondingly, denote by $V^{+}$the pair $(V,+)$ and by $V^{-}$the pair $(V,-)$. Then introduce the isomorphism of Heisenberg algebras $\theta$ defined by

$$
\theta V^{+}=V^{-} \theta V^{-}=V^{+}, \quad \forall V \in \mathfrak{n}_{0} .
$$

Clearly,

$$
\theta^{2}=I \theta J_{Z^{+}} V^{+}=\left(J_{Z} V\right)^{-}=J_{Z^{-}} V^{-}, \quad \forall Z \in \mathfrak{z} \forall V \in \mathfrak{v} .
$$

Let $\mathfrak{n}_{0+}=\mathfrak{z}_{+} \oplus \mathfrak{v}_{+}$, and $\mathfrak{n}_{0-}=\mathfrak{z}_{-} \oplus \mathfrak{v}_{-}$, where $\mathfrak{z}_{+}$and $\mathfrak{z}_{-}$are the centres of $\mathfrak{n}_{0+}$ and $\mathfrak{n}_{0-}$, and consider the decompositions

$$
\mathfrak{v}_{\sigma}=\mathfrak{v}_{11 \sigma} \oplus \mathfrak{v}_{21 \sigma} \oplus \mathfrak{v}_{22 \sigma} \oplus \mathfrak{v}_{12 \sigma}, \quad \text { where } \sigma \in\{+,-\},
$$

corresponding to (4.1.1).

\subsection{Construction of $\mathfrak{g}_{0}$}

Let $\mathscr{D}=\mathscr{D}\left(\mathfrak{n}_{0}\right)$ denote the space of derivations of $\mathfrak{n}_{0}$. The commutator $[\cdot, \cdot]$ provides $\mathscr{D}$ with a structure of Lie algebra. The operator $\Theta$ on $\mathscr{D}$, defined by

$$
\Theta D=-{ }^{t} D,
$$

where ${ }^{t}$ is the transpose of $D$, is an involution. We denote by $\mathcal{D}_{T}=\mathcal{D}_{T}\left(\mathfrak{n}_{0}\right)$ the subspace of $\mathscr{D}$ consiting of derivations acting trivially on the centre.

Proposition 4.2.1. If the linear map $D: \mathfrak{n}_{0} \rightarrow \mathfrak{n}_{0}$ is a derivation, then

$$
J_{\Theta D Z}=-J_{Z} D+(\Theta D) J_{Z}, \quad \forall Z \in \mathfrak{z} .
$$

In particular, if $D \in D_{T}$,

$$
\Theta D \in D_{T} J_{Z}(\Theta D)=D J_{Z}, \quad \forall Z \in \mathfrak{z},
$$

and $\mathfrak{D}_{T}$ is a subalgebra of $\mathfrak{D}$. 
Proof. If $D \in \mathcal{D}$, for all $Z \in \mathfrak{z}$ and $X, Y \in \mathfrak{v}$

$$
\begin{aligned}
\left\langle{ }^{t} D Z X, Y\right\rangle & =\left\langle{ }^{t} D Z,[X, Y]\right\rangle=\langle Z, D[X, Y]\rangle \\
& =\langle Z,[D X, Y]+[X, D Y]\rangle \\
& =\left\langle J_{Z} D X, Y\right\rangle+\left\langle{ }^{t} D J_{Z} X, Y\right\rangle,
\end{aligned}
$$

which implies

$$
{ }^{J^{t}} D Z=J_{Z} D+{ }^{t} D J_{Z}
$$

from which the first part of the statement follows. This clearly implies (4.2.2) for any $D \in \mathscr{D}_{T}$, from which it follows that $\mathscr{D}_{T}$ is an algebra.

To any derivation $D$ of $\mathfrak{n}_{0+}$ the rule

$$
U_{-} \mapsto-\theta\left({ }^{t} D U_{+}\right)
$$

associates a derivation of $\mathfrak{n}_{0-}$ which shall still be denoted by $D$. From (4.2.3) it follows that

$$
\theta\left(D U_{-}\right)=(\Theta D) \theta U_{-} .
$$

Accordingly we will first define a derivation on $\mathfrak{n}_{0+}$, and then extend it to $\mathfrak{n}_{0+} \oplus \mathfrak{n}_{0-}$. As a first example, consider the operator $H_{V}$ defined by

$$
\left.H_{V}\right|_{\mathfrak{z}_{+}}=2 I,\left.\quad H_{V}\right|_{\mathfrak{v}_{+}}=I,\left.\quad H_{V}\right|_{\mathfrak{v}_{-}}=-I,\left.\quad H_{V}\right|_{\mathfrak{z}_{-}}=-2 I,
$$

where $I$ is the identity and $\left.H\right|_{\mathfrak{u}}$ is the restriction of $H$ to the subspace $\mathfrak{u}$. Consider a linear map $E$ on $\mathfrak{n}_{0}$ defined by

$$
E Z=0, \quad E X_{0}=a Y_{0}, \quad E Y_{0}=b J Y_{0}, \quad E J Y_{0}=c J X_{0}, \quad E J X_{0}=0,
$$

$a, b, c \in \mathbb{R}$. Notice that $E$ is a derivation if and only if $c=-a$. From the second formula in (4.2.2) we get

$$
\Theta E Z=0, \Theta E X_{0}=0, \Theta E Y_{0}=-a X_{0}, \Theta E J Y_{0}=-b Y_{0}, E J X_{0}=a J Y_{0},
$$

from which, setting

$$
H_{T} \equiv[\Theta E, E]
$$

(which gives $\left.H_{T}\right|_{\mathfrak{v}}=E J E J-J E J E$ ), we deduce

$$
\left[H_{T}, E\right] X_{0}=\left(2 a^{2}-b^{2}\right) E X_{0}\left[H_{T}, E\right] Y_{0}=2\left(b^{2}-a^{2}\right) E Y_{0} .
$$

Therefore, $\left[H_{T}, E\right]=\lambda E$ if and only if $4 a^{2}=3 b^{2}$. Take $b=1$, then

$$
\mathfrak{g}_{T} \equiv \operatorname{span}\left\{E, \Theta E, H_{T}\right\}
$$


is a Lie algebra isomorphic to $\mathfrak{s t}(1,1)$. Clealry, $\Theta$ plays the rôle of the Cartan involution of $\mathfrak{g}_{T}$. We set $a=1$ and $b=\frac{2}{\sqrt{3}}$, obtaining

$$
E X_{0}=Y_{0}, \quad E Y_{0}=\frac{2}{\sqrt{3}} J Y_{0}, \quad E J Y_{0}=-J X_{0}, \quad E J X_{0}=0,
$$

which yield

$$
\begin{aligned}
2(\Theta E) X_{0} & =0, \quad(\Theta E) Y_{0}=-X_{0}, \\
(\Theta E) J Y_{0} & =-\frac{2}{\sqrt{3}} Y_{0}, \quad(\Theta E) J X_{0}=J Y_{0},
\end{aligned}
$$

and

$$
\left[H_{T}, E\right]=\frac{2}{3} E, \quad\left[H_{T}, \Theta E\right]=-\frac{2}{3} \Theta E .
$$

Furthermore,

$$
\left.H_{T}\right|_{\mathfrak{z}_{+}}=0
$$

and

$$
\left.H_{T}\right|_{\mathfrak{v}_{11+}}=-I,\left.\quad H_{T}\right|_{\mathfrak{v}_{12+}}=I,\left.\quad H_{T}\right|_{\mathfrak{v}_{21+}}=-\frac{1}{3} I,\left.\quad H_{T}\right|_{\mathfrak{v}_{22+}}=\frac{1}{3} I .
$$

On $\mathfrak{g}_{T}$ we introduce an inner product by setting

$$
\left\langle D_{1}, D_{2}\right\rangle \equiv \frac{3}{10} \operatorname{tr}\left(D_{1} \Theta D_{2}\right) .
$$

It is easy to see that $E, \Theta E$, and $H_{T}$ are mutually orthogonal, and that

$$
\langle E, E\rangle=\langle\Theta E, \Theta E\rangle=1\left\langle H_{T}, H_{T}\right\rangle=\frac{2}{3} .
$$

It is clear that $H_{V}$ and $H_{T}$ are symmetric derivations and that

$$
\left[H_{V}, E\right]=\left[H_{V}, \Theta E\right]=\left[H_{V}, H_{T}\right]=0 .
$$

Denote by $\mathfrak{a}$ the real span of $H_{V}$ and $H_{T}$, and set

$$
\mathfrak{g}_{0} \equiv \mathfrak{a}+\mathfrak{g}_{T}=\mathbb{R} H_{V} \oplus \mathfrak{g}_{T}
$$

Observe that $\mathfrak{g}_{0}$ is a Lie subalgebra of $\mathcal{D}$ and endow $\mathfrak{g}_{0} \oplus \mathfrak{n}_{0+}$ and $\mathfrak{g}_{0} \oplus \mathfrak{n}_{0-}$ with the structure of semidirect product of Lie algebras. Extend $\langle\cdot, \cdot\rangle$ to $\mathfrak{g}_{0}$ by setting

$$
\left\langle H_{V}, H_{V}\right\rangle=2\left\langle H_{V}, D\right\rangle=0, \quad \forall D \in \mathfrak{g}_{T} .
$$




\subsection{The algebra $\mathfrak{g}$}

Let

$$
\mathfrak{g}=\mathfrak{n}_{0-} \oplus\left(\mathfrak{a}+\mathfrak{g}_{T}\right) \oplus \mathfrak{n}_{0+},
$$

and $\langle\cdot, \cdot\rangle$ be the orthogonal sum of the given inner products on the single components. Extend $\theta$ to $\mathfrak{g}$ by putting

$$
\left.\theta\right|_{\mathfrak{g}_{0}}=\Theta
$$

It is now time to introduce the root spaces:

$$
\begin{array}{llll}
\mathfrak{g}_{\alpha}=\mathbb{R} E, & \mathfrak{g}_{-\alpha}=\mathbb{R} \theta E, & \mathfrak{g}_{2 \alpha+3 \beta}=\mathfrak{z}_{+}, & \mathfrak{g}_{-2 \alpha-3 \beta}=\mathfrak{z}_{-} \\
\mathfrak{g}_{\beta}=\mathfrak{v}_{11+}, & \mathfrak{g}_{\alpha+\beta}=\mathfrak{v}_{21+}, & \mathfrak{g}_{\alpha+2 \beta}=\mathfrak{v}_{22+}, & \mathfrak{g}_{\alpha+3 \beta}=\mathfrak{v}_{11+} \\
\mathfrak{g}_{-\beta}=\mathfrak{v}_{11-}, & \mathfrak{g}_{-\alpha-\beta}=\mathfrak{v}_{21-}, & \mathfrak{g}_{-\alpha-2 \beta}=\mathfrak{v}_{22-}, & \mathfrak{g}_{-\alpha-3 \beta}=\mathfrak{v}_{11-}
\end{array}
$$

The vectors $H_{V}, H_{T}, Z, X_{0}, Y_{0}, J X_{0}, J Y_{0}, \theta Z, \theta X_{0}, \theta Y_{0}, \theta J X_{0}, \theta J Y_{0}, E$, and $\theta E$ form a basis of $\mathfrak{g}$. The task at hand now is to write down the multiplication table in terms of this basis. Of course, some brackets have been already defined: indeed, we know the brackets in the semidirect product Lie algebras $\mathfrak{g}_{0} \oplus \mathfrak{n}_{0+}$ and $\mathfrak{g}_{0} \oplus \mathfrak{n}_{0-}$. If $Y \in \mathfrak{g}_{\gamma}$ and $Y^{\prime} \in \mathfrak{g}_{\delta}$, and $\gamma+\delta \notin \Delta$, we set $\left[Y, Y^{\prime}\right]=0$. We require that the bracket is skewsymmetric and satisfies

$$
\theta[U, V]=[\theta U, \theta V], \quad \forall U, V .
$$

Then, if $\gamma \in \Delta_{+}$and $Y \in \mathfrak{g}_{\gamma}$, we set

$$
\left[Y^{-}, Y\right]=[\theta Y, Y]=\frac{1}{2}(\gamma \mid 3 \alpha+2 \beta) H_{V}+\frac{3}{2}(\gamma \mid \alpha) H_{T} .
$$

For $Z \in \mathfrak{z}$ and $V \in \mathfrak{v}$, we define

$$
\left[Z^{+}, V^{-}\right]=J_{Z^{+}} V^{+},
$$

which by (4.3.1) and the second equation in (4.1.2) gives

$$
\left[Z^{-}, V^{+}\right]=J_{Z^{-}} V^{-} \text {. }
$$

Finally, we put

$$
E=\left[\theta X_{0}, Y_{0}\right]=\left[J X_{0}, \theta J Y_{0}\right]=\frac{\sqrt{3}}{2}\left[\theta Y_{0}, J Y_{0}\right],
$$

which as before provides

$$
\theta E=\left[X_{0}, \theta Y_{0}\right]=\left[\theta J X_{0}, J Y_{0}\right]=\frac{\sqrt{3}}{2}\left[Y_{0}, \theta J Y_{0}\right]
$$




\section{Some subalgebras of $\mathfrak{g}$}

Lemma 5.1.1. The linear map $\sigma$ defined by

$$
\left.\sigma\right|_{\mathfrak{z}}=\left.\sigma\right|_{\theta \mathfrak{z}}=I,\left.\quad \sigma\right|_{\mathfrak{v}}=J,\left.\quad \sigma\right|_{\theta \mathfrak{v}}=\theta \circ J \circ \theta,\left.\quad \sigma\right|_{\mathfrak{g}_{T}}=\theta,\left.\sigma\right|_{\mathbb{R} H_{V}}=-\theta I,
$$

satisfies

$$
\sigma[U, V]=[\sigma U, \sigma V], \quad \forall V, U \in \mathfrak{g},
$$

$$
\sigma \circ \theta=\theta \circ \sigma .
$$

Proof. It is clear from the definition that $\sigma$ commutes with $\theta$. The restriction of $\sigma$ to the Heisenberg algebra $\mathfrak{n}_{0}$ is an automorphism of Lie algebras since

$$
[\sigma U, \sigma J U]=\left[J U, J^{2} U\right]=-[J U, U]=Z=\sigma Z, \quad \forall Z \in \mathfrak{z} .
$$

The same holds for the restriction of $\sigma$ to $\theta \mathfrak{n}_{0}$ by (4.3.1) since $\theta$ is an invertible linear map from $\mathfrak{n}_{0}$ onto $\theta \mathfrak{n}_{0}$. Moreover, it is clear that the restriction of $\theta$, and hence of $\sigma$, to $\mathfrak{g}_{T}$ is an automorphism. Evidently $\sigma$ commutes with the action of $H_{V}$ on $\mathfrak{g}$. This, being $\sigma H_{V}=H_{V}$, implies that

$$
\sigma\left[H_{V}, U\right]=\left[\sigma H_{V}, \sigma U\right], \quad \forall U \in \mathfrak{g} .
$$

In particular, $\sigma$ preserves the decomposition of $\mathfrak{g}$ in eigenspaces of $H_{V}$. Hence, (5.1.1) is trivially satisfied for all $U \in \mathfrak{g}_{T}$ and $V \in \mathfrak{z} \oplus \theta \mathfrak{z}$. What remains is to prove that (5.1.1) follows by direct computations. From (5.1.2) and (4.3.5) we obtain

$$
\left[\sigma \theta X_{0}, \sigma Y_{0}\right]=\left[\theta J X_{0}, J Y_{0}\right]=\theta E=\sigma E,
$$

which by (4.3.4) yields

$$
\left[\sigma \theta X_{0}, \sigma Y_{0}\right]=\sigma E=\sigma\left[\theta X_{0}, Y_{0}\right]
$$

Similarly, we get

$$
\begin{aligned}
{\left[\sigma J X_{0}, \sigma \theta J Y_{0}\right] } & =\left[J^{2} X_{0}, \theta J^{2} Y_{0}\right]=\left[X_{0}, \theta Y_{0}\right] \\
& =\theta E=\sigma E=\sigma\left[\theta X_{0}, Y_{0}\right],
\end{aligned}
$$

and

$$
\begin{aligned}
\frac{\sqrt{3}}{2}\left[\sigma \theta Y_{0}, \sigma J Y_{0}\right] & =\frac{\sqrt{3}}{2}\left[\theta J Y_{0}, J^{2} Y_{0}\right]=\frac{\sqrt{3}}{2}\left[Y_{0}, \theta J Y_{0}\right] \\
& =\theta E=\sigma E=\frac{\sqrt{3}}{2} \sigma\left[\theta Y_{0}, J Y_{0}\right] .
\end{aligned}
$$

From these identities it follows, by (5.1.2), that

$$
\sigma \theta E=\left[\sigma X_{0}, \sigma \theta Y_{0}\right]=\left[\sigma \theta J X_{0}, \sigma J Y_{0}\right]=\frac{\sqrt{3}}{2}\left[\sigma Y_{0}, \sigma \theta J Y_{0}\right]
$$


To conclude the proof it is now enough to prove that $[\sigma U, \sigma V]=\sigma[U, V]$ for $U \in \mathfrak{g}_{T}$ and $V \in \mathfrak{v}$, since this formula will provide (5.1.1) for $U \in \mathfrak{g}_{T}$ and $V \in \theta \mathfrak{v}$ by (5.1.2). From (4.2.5) we deduce

$$
\left[\sigma E, \sigma X_{0}\right]=\left[\theta E, J X_{0}\right]=J Y_{0},
$$

which being, by (4.2.4),

$$
\sigma\left[E, X_{0}\right]=\sigma Y_{0}=J Y_{0}
$$

yields

$$
\sigma\left[E, X_{0}\right]=\left[\sigma E, \sigma X_{0}\right]
$$

We find, by (4.2.5),

$$
\left[\sigma E, \sigma Y_{0}\right]=\left[\theta E, J Y_{0}\right]=-\frac{2}{\sqrt{3}} Y_{0},
$$

which gives the desidered identity, being, by (4.2.4),

$$
\sigma\left[E, Y_{0}\right]=\frac{2}{\sqrt{3}} \sigma J Y_{0}=-\frac{2}{\sqrt{3}} Y_{0} .
$$

Similalrly, we get

$$
\begin{aligned}
{\left[\sigma E, \sigma J Y_{0}\right] } & =-\left[\theta E, Y_{0}\right]=X_{0}=-J^{2} X_{0} \\
& =-\sigma J X_{0}=\sigma\left[E, J Y_{0}\right]
\end{aligned}
$$

and

$$
\left[\sigma E, \sigma J X_{0}\right]=-\left[\theta E, X_{0}\right]=0=\sigma\left[E, J X_{0}\right]
$$

Proposition 5.1.2. Let $\gamma \in \Delta$, and $U \in \mathfrak{g}_{\gamma}$ a unit vector. Put $H_{\gamma}=[\theta U, U]$, then

$$
\mathfrak{g}_{(\gamma)}=\mathfrak{g}_{\gamma} \oplus\left(\mathbb{R} H_{\gamma}\right) \oplus \mathfrak{g}_{-\gamma}
$$

is a subalgebra of $\mathfrak{g}$ isomorphic to $\mathfrak{s l}(1,1)$.

Proof. It follows from (4.3.2) that

$$
\left[H_{\gamma}, U\right]=\frac{1}{2}\left[(\omega \mid \gamma) H_{V}+(\alpha \mid \gamma) H_{T}, U\right]=(\gamma \mid \gamma) U, \quad \forall U \in \mathfrak{g}_{\gamma} .
$$

and hence, the statement follows.

Let

$$
\Delta_{l+}=\{\beta, 3 \alpha+2 \beta, 3 \alpha+\beta\} \Delta_{l}=\Delta_{l+} \cup\left(-\Delta_{l+}\right) .
$$

Comparing the multiplication table introduced in this section with that defined in Sect. 5 of [2], we obtain the following proposition: 
Proposition 5.1.3. The subspace

$$
\mathfrak{h}=\sum_{\gamma \in \Delta_{l}} \oplus \mathfrak{g}_{\gamma}
$$

is a subalgebra of $\mathfrak{g}$ isomorphic to the Lie algebra $\mathfrak{s l}(3, \mathbb{R})$.

Let

$$
\begin{aligned}
\mathfrak{n}_{\beta} & =\mathfrak{g}_{-3 \alpha-\beta} \oplus \mathfrak{g}_{-\alpha} \oplus \mathfrak{g}_{\alpha+\beta} \oplus \mathfrak{g}_{3 \alpha+2 \beta} \oplus \mathfrak{g}_{\beta}, \\
\mathfrak{n}_{3 \alpha+\beta} & =\mathfrak{g}_{3 \alpha+2 \beta} \oplus \mathfrak{g}_{2 \alpha+\beta} \oplus \mathfrak{g}_{\alpha} \oplus \mathfrak{g}_{-\beta} \oplus \mathfrak{g}_{3 \alpha+\beta} .
\end{aligned}
$$

Proposition 5.1.4. The subspaces $\mathfrak{n}_{\beta} \oplus\left(\mathfrak{g}_{(2 \alpha+\beta)}+\mathfrak{a}\right), \mathfrak{n}_{3 \alpha+\beta} \oplus\left(\mathfrak{g}_{(\alpha+\beta)}+\mathfrak{a}\right), \theta \mathfrak{n}_{\beta} \oplus$ $\left(\mathfrak{g}_{(2 \alpha+\beta)}+\mathfrak{a}\right), \theta \mathfrak{n}_{3 \alpha+\beta} \oplus\left(\mathfrak{g}_{(\alpha+\beta)}+\mathfrak{a}\right)$, and $\theta \mathfrak{n}_{0} \oplus\left(\mathfrak{g}_{T}+\mathfrak{a}\right)$ are Lie algebras isomorphic to $\mathfrak{n}_{0} \oplus\left(\mathfrak{g}_{T}+\mathfrak{a}\right)$.

Proof. $\mathfrak{n}_{0} \oplus\left(\mathfrak{g}_{T}+\mathfrak{a}\right)$ and $\theta \mathfrak{n}_{0} \oplus\left(\mathfrak{g}_{T}+\mathfrak{a}\right)$ are semidirect products of Lie algebras, and $\theta$ is, by (4.3.1), an isomorphism of Lie algebras between them. We show that $\mathfrak{n}_{\beta} \oplus\left(\mathfrak{g}_{(2 \alpha+\beta)}+\mathfrak{a}\right)$ is isomorphic to $\mathfrak{n}_{0} \oplus\left(\mathfrak{g}_{T}+\mathfrak{a}\right)$. From this it follows by (4.3.1), that $\theta \mathfrak{n}_{\beta} \oplus\left(\mathfrak{g}_{(2 \alpha+\beta)}+\mathfrak{a}\right)$ is isomorphic to $\mathfrak{n}_{0} \oplus\left(\mathfrak{g}_{T}+\mathfrak{a}\right)$. Similalrly, $\mathfrak{n}_{3 \alpha+\beta} \oplus$ $\left(\mathfrak{g}_{(\alpha+\beta)}+\mathfrak{a}\right)$ is, by (5.1.1), isomorphic to $\mathfrak{n}_{\beta} \oplus\left(\mathfrak{g}_{(2 \alpha+\beta)}+\mathfrak{a}\right)$, and hence to $\mathfrak{n}_{0} \oplus$ $\left(\mathfrak{g}_{T}+\mathfrak{a}\right)$. This finally implies that $\theta \mathfrak{n}_{3 \alpha+\beta} \oplus\left(\mathfrak{g}_{(\alpha+\beta)}+\mathfrak{a}\right)$ is isomorphic to $\mathfrak{n}_{0} \oplus$ $\left(\mathfrak{g}_{T}+\mathfrak{a}\right)$.

We prove that $\mathfrak{n}_{\beta} \oplus\left(\mathfrak{g}_{(2 \alpha+\beta)}+\mathfrak{a}\right)$ is isomorphic to $\mathfrak{n}_{0} \oplus\left(\mathfrak{g}_{T}+\mathfrak{a}\right)$, showing that these two algebras have the same constants of structure. To make this evident we relabel the vectors in the basis of $\mathfrak{g}$, setting

$$
Z_{1} \equiv X_{0}, \quad X_{1} \equiv \theta J X_{0}, Y_{1} \equiv \theta E .
$$

Let

$$
\mathfrak{v}_{(\beta)} \equiv \mathfrak{g}_{-3 \alpha-\beta} \oplus \mathfrak{g}_{-\alpha} \oplus \mathfrak{g}_{\alpha+\beta} \oplus \mathfrak{g}_{3 \alpha+2 \beta},
$$

and define

$$
J^{(\beta)} U \equiv\left[Z_{1}, \theta U\right], \quad \text { for } U \in \mathfrak{v}_{(\beta)} .
$$

We have, by (3.1.5) and (4.2.5),

$$
\begin{gathered}
J^{(\beta)} X_{1}=\left[Z_{1}, \theta X_{1}\right]=\left[X_{0}, J X_{0}\right]=Z, \\
J^{(\beta)} Y_{1}=\left[Z_{1}, \theta Y_{1}\right]=\left[X_{0}, E\right]=-Y_{0},
\end{gathered}
$$

from which we get

$$
\left[X_{1}, J^{(\beta)} X_{1}\right]=\left[\theta J X_{0}, Z\right]=X_{0}=Z_{1}
$$

by (3.1.1) and (3.1.2),

$$
\left[Y_{1}, J^{(\beta)} Y_{1}\right]=-\left[\theta E, Y_{0}\right]=X_{0}=Z_{1}
$$


by (4.2.4),

$$
\left[X_{1}, J^{(\beta)} Y_{1}\right]=-\left[\theta J X_{0}, Y_{0}\right]=0,\left[Y_{1}, J^{(\beta)} X_{1}\right]=[\theta E, Z]=0 .
$$

Hence, $\mathfrak{n}_{\beta}$ is isomorphic to $\mathfrak{n}_{0}$.

The algebra $\mathfrak{g}_{(\alpha+\beta)}$ is, by Proposition 5.1.2, isomorphic to $\mathfrak{g}_{T}$. Thus, $\mathfrak{g}_{(\alpha+\beta)}+\mathfrak{a}$ is isomorphic to $\mathfrak{g}_{T}+\mathfrak{a}$. Set $E_{1} \equiv\left[\theta X_{1}, Y_{1}\right]$, plugging the last two relations in (5.1.3) into this formula and using the fourth in (4.2.4), we get

$$
E_{1}=\left[\theta X_{1}, Y_{1}\right]=\left[J X_{0}, \theta E\right]=-J Y_{0} .
$$

We show that

$$
\begin{array}{ll}
{\left[E_{1}, X_{1}\right]=Y_{1},} & {\left[E_{1}, Y_{1}\right]=\frac{2}{\sqrt{3}} J^{(\beta)} Y_{1},} \\
{\left[E_{1}, J^{(\beta)} Y_{1}\right]=-J^{(\beta)} X_{1},} & {\left[E_{1}, J^{(\beta)} Y_{1}\right]=0 .}
\end{array}
$$

From (5.1.6) and the first equation in (4.3.5), it follows that

$$
\left[E_{1}, X_{1}\right]=-\left[J Y_{0}, \theta J X_{0}\right]=-\theta E=Y_{1}
$$

Similalrly, from (5.1.6), we deduce

$$
\left[E_{1}, Y_{1}\right]=-\left[J Y_{0}, \theta E\right]=\frac{2}{\sqrt{3}} Y_{0}=\frac{2}{\sqrt{3}} J^{(\beta)} Y_{1}
$$

by the third equation in (4.2.5),

$$
\left[E_{1}, J^{(\beta)} Y_{1}\right]=-\left[J Y_{0}, Y_{0}\right]=-Z=-J^{(\beta)} X_{1}
$$

by (5.1.5) and (3.1.5), and finally,

$$
\left[E_{1}, J^{(\beta)} X_{1}\right]=-\left[J Y_{0}, Z\right]=0
$$

by (5.1.4), and (4.3.5). The relations analogous to the (5.1.7)s with $\theta E_{1}$ in place of $E_{1}$ may be proved similalrly. Now the statement follows.

\section{The jacobi identity for $\mathfrak{g}$}

Theorem 6.1. $\mathfrak{g}$ is a simple Lie algebra.

Proof. We will prove that $\mathfrak{g}$ is a Lie algebra, showing that the Jacobi identity holds. In Sect. 5 we showed that many subspaces of $\mathfrak{g}$ are indeed Lie algebras, thus, few Jacobi identities remain to be proved. Using the linear maps $\theta$ and $\sigma$ which satisfy (4.3.1) and (5.4.1), the number of identities which must be proved is further reduced to the relations involving triples of vectors lying in (1) $\mathfrak{g}_{\alpha}, \mathfrak{g}_{\alpha+\beta}$, and $\mathfrak{g}_{-2 \alpha-\beta}$, (2) $\mathfrak{g}_{\omega}, \mathfrak{g}_{-\alpha-\beta}$, and $\mathfrak{g}_{-2 \alpha-\beta}$, and (3) $\mathfrak{g}_{\omega}, \mathfrak{g}_{-\alpha-\beta}$, and $\mathfrak{g}_{\alpha}$. 
For (1), since $\left[\theta J Y_{0}, Y_{0}\right]=-(\sqrt{3} / 2) \theta E$ by the second relation in (4.3.5), (4.3.2) yields

$$
\left[\left[\theta J Y_{0}, Y_{0}\right], E\right]=-\frac{2}{\sqrt{3}}[\theta E, E]=-\frac{2}{\sqrt{3}} H_{T} .
$$

By the second equation in (4.2.4) and (4.3.2), we get

$$
\left[\left[Y_{0}, E\right], \theta J Y_{0}\right]=-\frac{2}{\sqrt{3}}\left[J Y_{0}, \theta J Y_{0}\right]=\frac{1}{\sqrt{3}} H_{V}+\frac{1}{\sqrt{3}} H_{T},
$$

and similalrly, by the third equation in (4.2.5) and (4.3.2),

$$
\left[\left[E, \theta J Y_{0}\right], Y_{0}\right]=-\frac{2}{\sqrt{3}}\left[\theta Y_{0}, Y_{0}\right]=-\frac{1}{\sqrt{3}} H_{V}+\frac{1}{\sqrt{3}} H_{T} .
$$

The sum of these three formulas is zero, and the result follows.

For (2), (3.1.5) and (4.3.2) give

$$
\left[\left[\theta J Y_{0}, \theta Y_{0}\right], Z\right]=-[\theta Z, Z]=-H_{V} .
$$

Since, by (4.3.3),

$$
\left[Z, \theta Y_{0}\right]=J Y_{0}\left[Z, \theta J Y_{0}\right]=J^{2} Y_{0}=-Y_{0},
$$

we find, by (4.3.2),

$$
\left[\left[\theta Y_{0}, Z\right], \theta J Y_{0}\right]=\frac{1}{2} H_{V}+\frac{1}{2} H_{T}\left[\left[Z, \theta J Y_{0}\right], \theta Y_{0}\right]=-\frac{1}{2} H_{T}+\frac{1}{2} H_{V} .
$$

Finally for (3), the second equation in (5.2.5) and (4.3.3) provide

$$
\left[\left[\theta Y_{0}, E\right], Z\right]=\left[\theta X_{0}, Z\right]=-J X_{0},
$$

(4.3.3) and the third in (4.2.5) give

$$
\left[\left[Z, \theta Y_{0}\right], E\right]=\left[J Y_{0}, E\right]=J X_{0},
$$

from which, being $[Z, E]=0$, we obtain the Jacobi identity.

Now we prove that $\mathfrak{g}$ is simple. Suppose $\mathfrak{h}$ is a nontrivial ideal in $\mathfrak{g}$ and let $U \in \mathfrak{h}$ be nonzero. Since $\operatorname{ad} Z$ is nilpotent there is a nonnegative integer $k$ such that

$$
(\operatorname{ad} Z)^{k+1} U=0 V=(\operatorname{ad} Z)^{k} U \neq 0 .
$$

Being $[Z, V]=0$, it follows that $V \in\left(\mathfrak{n}_{0} \oplus \mathfrak{g}_{T}\right) \cap \mathfrak{h}$. Hence

$$
V=a_{1} X_{0}+a_{2} Y_{0}+a_{3} J Y_{0}+a_{4} J X_{0}+a_{5} Z+a_{6} E+a_{7} \theta E+a_{8} H_{T},
$$

where $a_{1}, a_{2}, a_{3}, a_{4}, a_{5}, a_{6}, a_{7}, a_{8}$ are real numbers. It is easy to see that

$$
\operatorname{span}\left\{\left[V, H_{\gamma}\right]: \gamma=\alpha,-\alpha, \beta, \alpha+\beta, 2 \alpha+\beta, 3 \alpha+\beta, 3 \alpha+2 \beta\right\} \subset \mathfrak{h}
$$

has dimension given by the number of nonzero coefficients among $a_{1}, a_{2}, a_{3}, a_{4}$, $a_{5}, a_{6}, a_{7}$. It follows, therefore, that there is a nonvanishing $W \in \mathfrak{h}$ lying in some 
root space $\mathfrak{g}_{\gamma}$. Thus, $\mathfrak{g}_{\gamma} \subset \mathfrak{h}$, and by Corollary 4.2 of [2] all the root spaces are contained in $\mathfrak{h}$, which implies $\mathfrak{h}=\mathfrak{g}$.

Now we prove that there is only one simple Lie algebra with root system $G_{2}$ and dimension 14, i.e. $d_{\gamma}=1$ for all $\gamma \in \Delta$. Let $\mathfrak{l}$ be such a Lie algebra, and let $Z \in \mathfrak{l}_{\omega}, X \in \mathfrak{l}_{\beta}$, and $Y \in \mathfrak{l}_{\alpha+\beta}$ be unit vectors. Using the Jacobi identity and the geometry of the root system, we easily deduce from $E=[\theta X, Y]$ the following relations:

$$
\begin{array}{ll}
{[E, X]=Y,} & {[\theta E, J X]=J Y,} \\
{[\theta E, Y]=-X,} & {[E, J Y]=-J X}
\end{array}
$$

which, since $\theta$ is an automorphism, provide

$$
\begin{array}{ll}
{[\theta E, \theta X]=\theta Y,} & {[E, \theta J X]=\theta J Y,} \\
{[E, \theta Y]=-\theta X,} & {[\theta E, \theta J Y]=-\theta J X .}
\end{array}
$$

We also have

$$
\begin{aligned}
& {[[\theta E, E], X]=(\alpha \mid \beta) X=-X,} \\
& {[[\theta E, E], Y]=(\alpha \mid \alpha+\beta) Y=-\frac{1}{3} Y,} \\
& {[[\theta E, E], J Y]=(\alpha \mid 2 \alpha+\beta) J Y=\frac{1}{3} J Y,} \\
& {[[\theta E, E], J X]=(\alpha \mid 3 \alpha+\beta) J X=J X .}
\end{aligned}
$$

Since $d_{\gamma}=1$ for all $\gamma \in \Delta$, we put

$$
[E, Y]=[[\theta X, Y], Y]=a J Y, \quad \text { with } a \in \mathbb{R},
$$

from which, using the Jacobi identity, the third equation in (6.1.3), and the definition of $E$, we deduce

$$
\begin{aligned}
-\frac{1}{3} E & =(\alpha+\beta \mid \alpha) E=[[\theta Y, Y], E] \\
& =[[E, Y], \theta Y]+[[\theta Y, E], Y] \\
& =a[J Y, \theta Y]+[\theta X, Y] \\
& =a[J Y, \theta Y]+E,
\end{aligned}
$$

that is,

$$
a[\theta Y, J Y]=\frac{4}{3} E .
$$

From this relation, by Lemma 3.3.4, it follows that

$$
\left(\frac{4}{3}\right)^{2}=\left(\frac{4}{3}\right)^{2}\|E\|^{2}=a^{2}\|[\theta Y, J Y]\|^{2}=\frac{4}{3} a^{2} .
$$


Hence, $a= \pm \frac{2}{\sqrt{3}}$. The arbitrariness in the sign of $a$ is a consequence of the arbitrariness of the versus of $Z$, which is defined as a vector of unit norm in $\mathfrak{l}_{\omega}$. To fix the ideas we assume $a=\frac{2}{\sqrt{3}}$, which means, by (6.1.4).

$$
E=\frac{\sqrt{3}}{2}[\theta Y, J Y]
$$

and implies

$$
[E, Y]=[[\theta X, Y], Y]=\frac{2}{\sqrt{3}} J Y .
$$

All the other brackets can be now easily deduced from these, ones employing the Jacobi identity and the results of Sect. 3. For instance, from the definition of $J$ we obtain, using the Jacobi identity,

$$
[\theta E, J Y]=[\theta E,[Z, \theta Y]]=[\theta Y,[Z, \theta E]]+[Z,[\theta E, \theta Y]],
$$

which, being $(\omega \mid \alpha)=0$, by (6.1.6) and (3.1.5), implies

$$
[\theta E, J Y]=[Z, \theta[E, Y]]=\frac{2}{\sqrt{3}}[Z, \theta J Y]=-\frac{2}{\sqrt{3}} Y .
$$

Hence, $\mathfrak{l}$ and $\mathfrak{g}$, having the same constants of structure, are isomorphic.

\section{The complexification of $\mathfrak{g}$}

Lemma 7.1.1. Let

$$
\mathfrak{g}_{(\gamma)}=\mathfrak{g}_{-\gamma} \oplus(\mathfrak{m} \oplus \mathfrak{a}) \oplus \mathfrak{g}_{\gamma}
$$

be a real rank one simple Lie algebra with $d_{\gamma}=2$, and let $H_{\gamma} \in \mathfrak{a}$ and $M_{\gamma} \in \mathfrak{m}$ satisfy

$$
\left\|H_{\gamma}\right\|^{2}=\left\|M_{\gamma}\right\|^{2}=\gamma\left(H_{\gamma}\right)=\langle\gamma, \gamma\rangle \text {. }
$$

Define a linear map $\mathfrak{g}: \mathfrak{g}_{(\gamma)} \rightarrow \mathfrak{g}_{(\gamma)}$ by

$g: Z \mapsto \frac{1}{\langle\gamma, \gamma\rangle}\left(a d M_{\gamma}\right) Z, \quad g: \theta Z \mapsto-\frac{1}{\langle\gamma, \gamma\rangle} \theta\left(a d M_{\gamma}\right) Z, \quad$ for $Z$ in $\mathfrak{g}_{\gamma}$,

and by

$$
\text { g: } H_{\gamma} \mapsto M_{\gamma}, \quad \text { g }: M_{\gamma} \mapsto-H_{\gamma}
$$

Then $\mathrm{g}$ is a complex structure on $\mathfrak{g}$, i.e.

$$
\mathscr{g}^{2}=-I \mathscr{g}[U, V]=[\mathscr{g} U, V]=[U, \mathscr{g} V], \quad \forall U, V \in \mathfrak{g}_{(\gamma)} .
$$

We omit the proof which consists of a straightforward computation. 
Theorem 7.1.2. There exists one, and only one, simple Lie algebra with root system $G_{2}$ and dimension 28 , i.e. the complexification $\mathfrak{g}_{\mathbb{C}}$ of $\mathfrak{g}$.

Proof. By Theorem $6.1 \mathfrak{g}$ is a Lie algebra, hence, its complexification is a Lie algebra [5, p. 178-179]. Since $\mathfrak{g}$ is simple, $\mathfrak{g}_{\mathbb{C}}$ is semisimple by Cartan criterion for semisimplicity (see [5, p. 180]) and has root system of type $G_{2}$. In particular, there exists a semisimple Lie algebra with root system $G_{2}$ and dimension 28 .

Let $\mathfrak{h}$ be a semisimple Lie algebra with root system $G_{2}$ and multiplicities two, we shall prove that $\mathfrak{h}$ is the complexification of a Lie algebra isomorphic to $\mathfrak{g}$. This shows that this class of Lie algebras contains just one element. We know that the subalgebras $\mathfrak{n}$ and $\theta \mathfrak{n}$ of $\mathfrak{h}$ defined in Subsect. 3.1.1 are Heisenberg-type Lie algebras. Denote, as before, by $\mathfrak{g}_{\omega}$ the centre of $\mathfrak{n}$.

Take an orthonormal basis $\left\{Z_{1}, Z_{2}\right\}$ of $\mathfrak{g}_{\omega}$, a unit vector $X \in \mathfrak{g}_{\beta}$, and a unit vector $Y \in \mathfrak{g}_{\alpha+\beta}$, and set

$$
E_{1}=[\theta X, Y] E_{2}=\left[\theta X, J_{1} J_{2} Y\right]
$$

Then $E_{2}=-\left[\theta J_{1} J_{2} X, Y\right]$, by (3.3.9), and $\left\{E_{1}, E_{2}\right\}$ is an orthonormal basis of $\mathfrak{g}_{\alpha}$. Also set

$$
\begin{array}{ll}
H_{\omega}=\left[\theta Z_{1}, Z_{1}\right]=\left[\theta Z_{2}, Z_{2}\right], & M_{\omega}=\left[Z_{1}, \theta Z_{2}\right], \\
H_{\alpha}=\left[\theta E_{1}, E_{1}\right]=\left[\theta E_{2}, E_{2}\right], & M_{\alpha}=\left[E_{1}, \theta E_{2}\right] .
\end{array}
$$

Then define a linear map $\mathfrak{g}: \mathfrak{h} \rightarrow \mathfrak{h}$ by

$$
g \circ \theta+\theta \circ g=0
$$

$$
\begin{array}{ll}
\text { g: } Z \mapsto \frac{1}{2} \operatorname{ad} M_{\omega} Z & \text { for } Z \text { in } \mathfrak{g}_{\omega}, \\
\text { g: } U \mapsto \operatorname{ad} M_{\omega} U & \text { for } U \text { in } \mathfrak{v}, \\
\text { g: } E \mapsto \frac{3}{2} \operatorname{ad} M_{\alpha} E & \text { for } E \text { in } \mathfrak{g}_{\alpha},
\end{array}
$$

and

$$
\begin{array}{ll}
\text { g : } H_{\omega} \mapsto M_{\omega}, & \text { g : } M_{\omega} \mapsto-H_{\omega}, \\
\text { g : } H_{\alpha} \mapsto M_{\alpha}, & \text { g }: M_{\alpha} \mapsto-H_{\alpha} .
\end{array}
$$

Clearly $g^{2}=-I$, we must show $g[U, V]=[g U, V]=[U, g V]$. We start proving that for all $\gamma \in \Delta$ the restriction of $\mathcal{g}$ to the subalgebra $\mathfrak{g}_{(\gamma)}$ generated by $\mathfrak{g}_{\gamma}$ and $\mathfrak{g}_{-\gamma}$ is the complex structure introduced in Lemma 7.1.1, which by (7.1.4), shows

$$
\mathscr{g} V=\frac{1}{\langle\gamma, \gamma\rangle} \operatorname{ad}\left(M_{\gamma}\right) V, \quad \forall V \in \mathfrak{g}_{\gamma}
$$


This is obvious when $\gamma= \pm \omega$, or $\gamma= \pm \alpha$. For the other root spaces it is a consequence of the following relations which hold for all $Z \in \mathfrak{g}_{\omega}, X \in \mathfrak{g}_{\beta}$, and $Y \in \mathfrak{g}_{\alpha+\beta}$,

$$
\begin{aligned}
\operatorname{ad}\left(\left[Z_{1}, \theta Z_{2}\right]\right) X & =J_{1} J_{2} X=\frac{1}{2} \operatorname{ad}\left(\left[X, \theta J_{1} J_{2} X\right]\right) X, \\
\operatorname{ad}\left(\left[Z_{1}, \theta Z_{2}\right]\right) J_{Z} X & =J_{1} J_{2} J_{Z} X=\frac{1}{2} \operatorname{ad}\left(\left[J_{1} X, \theta J_{2} X\right]\right) J_{Z} X, \\
\operatorname{ad}\left(\left[Z_{1}, \theta Z_{2}\right]\right) Y & =J_{1} J_{2} Y=\frac{3}{2} \operatorname{ad}\left(\left[Y, \theta J_{1} J_{2} Y\right]\right) Y, \\
\operatorname{ad}\left(\left[Z_{1}, \theta Z_{2}\right]\right) J_{Z} Y & =J_{1} J_{2} J_{Z} Y=\frac{3}{2} \operatorname{ad}\left(\left[J_{1} X, \theta J_{2} X\right]\right) J_{Z} Y .
\end{aligned}
$$

Let $\gamma, \delta \in \Delta$ and $V \in \mathfrak{g}_{\gamma}$, we show that $\mathcal{g}\left[H_{\delta}, V\right]=\left[\mathcal{g} H_{\delta}, V\right]=\left[H_{\delta}, \mathscr{g} V\right]$. Since $\left[H_{\delta}, V\right] \in \mathfrak{g}_{\gamma}$, we have, by (7.1.5),

$$
\mathcal{g}\left[H_{\delta}, V\right]=\frac{1}{\langle\gamma, \gamma\rangle}\left[M_{\gamma},\left[H_{\delta}, V\right]\right],
$$

which, recalling that the action of $\mathfrak{m}$ preserves the root spaces, yields by Jacobi and (7.1.5),

$$
\begin{aligned}
g\left[H_{\delta}, V\right] & =\frac{1}{\langle\gamma, \gamma\rangle}\left[M_{\gamma},\left[H_{\delta}, V\right]\right]=\frac{1}{\langle\gamma, \gamma\rangle}\left[H_{\delta},\left[M_{\gamma}, V\right]\right] \\
& =\frac{\langle\delta, \gamma\rangle}{\langle\gamma, \gamma\rangle}\left[M_{\gamma}, V\right]=\langle\delta, \gamma\rangle \mathcal{g} V=\left[H_{\delta}, \mathcal{g} V\right] .
\end{aligned}
$$

Since, by (3.3.6),

$$
\left[M_{\delta}, V\right]=\frac{\langle\delta, \gamma\rangle}{\langle\gamma, \gamma\rangle}\left[M_{\gamma}, V\right]
$$

we get, by (7.1.4),

$$
\left[g H_{\delta}, V\right]=\left[M_{\delta}, V\right]=\frac{\langle\delta, \gamma\rangle}{\langle\gamma, \gamma\rangle}\left[M_{\gamma}, V\right]=\langle\delta, \gamma\rangle g V=\left[H_{\delta}, g V\right],
$$

from which the result follows. We now prove that $\mathcal{g}\left[M_{\delta}, V\right]=\left[\mathscr{g} M_{\delta}, V\right]=$ $\left[M_{\delta}, \mathcal{g} V\right]$. Since the action of $\mathfrak{m}$ preserves the root spaces, we have, by (7.1.5),

$$
g\left[M_{\delta}, V\right]=\frac{1}{\langle\gamma, \gamma\rangle}\left[M_{\gamma},\left[M_{\delta}, V\right]\right],
$$

which, by Jacobi, yields

$$
\mathscr{g}\left[M_{\delta}, V\right]=\frac{1}{\langle\gamma, \gamma\rangle}\left[M_{\delta},\left[M_{\gamma}, V\right]\right]=\left[M_{\delta}, \mathscr{g} V\right] .
$$

Moreover, being

$$
\left[M_{\gamma},\left[M_{\gamma}, V\right]\right]=-\langle\gamma, \gamma\rangle^{2} V,
$$


we get, by (3.3.6),

$$
\mathscr{g}\left[M_{\delta}, V\right]=\frac{1}{\langle\gamma, \gamma\rangle}\left[M_{\gamma},\left[M_{\delta}, V\right]\right]=\frac{\langle\delta, \gamma\rangle}{\langle\gamma, \gamma\rangle^{2}}\left[M_{\gamma},\left[M_{\gamma}, V\right]\right]=-\langle\delta, \gamma\rangle V,
$$

which provides the result, since

$$
\left[\mathcal{g} M_{\delta}, V\right]=-\left[H_{\delta}, V\right]=-\langle\delta, \gamma\rangle V .
$$

Since $\mathcal{g}$ preserves the root spaces, the second relation in (7.1.1) is clear for vectors in root spaces corresponding to pairs of roots with sum not in $\Delta$. Let $U, V \in \mathfrak{v}$ and $[U, V]=Z$. Write $Z=a Z_{1}+b Z_{2}$, then

$$
\begin{aligned}
\left\langle Z_{1},\left[J_{1} J_{2} U, V\right]\right\rangle & =\left\langle J_{1}^{2} J_{2} U, V\right\rangle=-\left\langle J_{2} U, V\right\rangle=-\left\langle Z_{2},[U, V]\right\rangle \\
& =-\left\langle Z_{2}, Z\right\rangle=-b,
\end{aligned}
$$

and, similarly,

$$
\left\langle Z_{2},\left[J_{1} J_{2} U, V\right]\right\rangle=\left\langle J_{2} J_{1} J_{2} U, V\right\rangle=\left\langle J_{1} U, V\right\rangle=\left\langle Z_{1},[U, V]\right\rangle=\left\langle Z_{1}, Z\right\rangle=a,
$$

which together imply

$$
[\mathscr{g} U, V]=\left[J_{1} J_{2} U, V\right]=a Z_{2}-b Z_{1} .
$$

Being

$$
\begin{aligned}
\mathscr{g}[U, V] & =\frac{1}{2} \operatorname{ad}\left(\left[Z_{1}, \theta Z_{2}\right]\right)[U, V]=\frac{1}{2}\left[\left[Z_{1}, \theta Z_{2}\right], Z\right] \\
& =a Z_{2}-b Z_{1},
\end{aligned}
$$

we find

$$
\mathscr{g}[U, V]=[\mathscr{g} U, V]
$$

which, since $U$ and $V$ are arbitrary, implies $\mathscr{g}[U, V]=[U, \mathfrak{g} V]$. Let $U \in \mathfrak{v}$ and $Z=a Z_{1}+b Z_{2} \in \mathfrak{g}_{\omega}$. The definition of $\mathscr{g}$ yields

$$
\begin{gathered}
\mathcal{g}[Z, \theta U]=J_{1} J_{2}[Z, \theta U]=J_{1} J_{2}\left(a J_{1} U+b J_{2} U\right)=a J_{2} U-b J_{1} U, \\
{[Z, g \theta U]=-\left[Z, \theta J_{1} J_{2} U\right]=-a J_{1}^{2} J_{2} U-b J_{2} J_{1} J_{2} U=a J_{2} U-b J_{1} U,}
\end{gathered}
$$

and

$$
[g Z, \theta U]=\frac{1}{2}\left[\left[\left[Z_{1}, \theta Z_{2}\right], Z\right], \theta U\right]=\frac{1}{2}\left[a Z_{2}-b Z_{1}, \theta U\right]=a J_{2} U-b J_{1} U,
$$

from which we deduce the second of the (7.1.1)'s with $Z$ in place of $U$ and $\theta U$ in 
place of $V$. Let $U \in \mathfrak{v}$ and $E=a E_{1}+b E_{2} \in \mathfrak{g}_{\alpha}$. Since on $\mathfrak{v}$

$$
J_{1} J_{2} \circ \operatorname{ad} E=\operatorname{ad} E \circ J_{1} J_{2} \operatorname{ad} E_{2}=J_{1} J_{2} \circ \operatorname{ad} E_{1},
$$

we obtain

$$
\mathcal{g}[E, U]=J_{1} J_{2}[E, U]=\left[E, J_{1} J_{2} U\right]=[E, \mathcal{g} U]
$$

and

$$
\begin{aligned}
\mathscr{g}[E, U] & =J_{1} J_{2}\left[a E_{1}+b E_{2}, U\right]=a J_{1} J_{2}\left[E_{1}, U\right]+b\left(J_{1} J_{2}\right)^{2}\left[E_{1}, U\right] \\
& =a\left[E_{2}, U\right]-b\left[E_{1}, U\right] .
\end{aligned}
$$

Since by definition of $g$

$$
[g E, U]=\frac{3}{2}\left[\left[\left[E_{1}, \theta E_{2}\right], E\right], U\right]=\left[a E_{2}-b E_{1}, U\right],
$$

we have the result. Similalrly, we might prove the second in (7.1.1) for $\theta E$ and $U$. Now (7.1.2) yields the result.

We have proved that $\mathfrak{h}$ has a complex structure $\boldsymbol{g}$. Therefore, there is a subalgebra $\mathfrak{g}_{0}$ such that

$$
\mathfrak{h}=\mathfrak{g}_{0} \oplus \mathfrak{g}^{\mathfrak{g}_{0}} .
$$

Clearly, $\mathfrak{g}_{0}$ has root system $G_{2}$ and $\operatorname{dim} \mathfrak{g}_{0}=14$. Since by Theorem 6.1 there is up to isomorphisms only one Lie algebra belonging to this class, $\mathfrak{g}_{0} \simeq \mathfrak{g}$.

Finally, the argument used in the proof of Theorem 6.1 to show that $\mathfrak{g}$ is simple works also here, and $\mathfrak{g}_{\mathbb{C}}$ is simple.

\section{References}

1. Bourbaki, N.: Groupes et Algèbre de Lie. Chaps. 4, 5 et 6, Paris: Masson 1981

2. Ciatti, P.: A Clifford algebra approach to simple Lie algebras of real rank two, I: the $A_{2}$ case. J. Lie Theory 10, 53-80 (2000)

3. Cowling, M., Dooley, A.: Korányi, A., Ricci, F.: H-type groups and Iwasawa decomposition. Adv. Math. 87, 1-4 (1997)

4. Cowling, M., Dooley, A., Korányi, A., Ricci, F: An approach to symmetric spaces of rank one via groups of Heisenberg type. J. Geom. Anal. 8(2), 199-237 (1998)

5. Helgason, S.: Differential Geometry, Lie Groups, and Symmetric Spaces. New York: Academic Press 1978

6. Kaplan, A.: Fundamental solutions for a class of hypoelliptic PDE generated by compositions of quadratic forms. Trans. Amer. Math. Soc. 258, 147-153 (1980)

7. Korányi, A: Geometric properties of Heisenberg type groups. Adv. Math. 56, 28-38 (1986)

8. Lawson, H., Michelson, M.L.: Spin Geometry. Princeton: Princeton University Press 1989 